\title{
Protée
}

\section{"Tout leur art est pure grimace ". Le personnage du médecin dans l'oeuvre de Wilson}

\section{Sharon Aronson-Lehavi}

Volume 27, numéro 1, 1999

La Mort de Molière et des autres

URI : https://id.erudit.org/iderudit/030546ar

DOI : https://doi.org/10.7202/030546ar

Aller au sommaire du numéro

Éditeur(s)

Département des arts et lettres - Université du Québec à Chicoutimi

ISSN

0300-3523 (imprimé)

1708-2307 (numérique)

Découvrir la revue

Citer cet article

Aronson-Lehavi, S. (1999). « Tout leur art est pure grimace ». Le personnage du médecin dans l'oeuvre de Wilson. Protée, 27(1), 81-86.

https://doi.org/10.7202/030546ar
Résumé de l'article

Cet article porte sur le personnage du docteur dans La Mort de Molière de Robert Wilson et de Heiner Müller. Mon argument principal est que toutes ses apparitions dans la vidéo sont de complexes citations de ce personnage de Molière qu'est Sganarelle, qui s'habille en docteur dans de nombreuses pièces pour porter un regard critique sur cette profession. C'est un des procédés ironiques que Wilson utilise pour montrer l'impuissance de la médecine à aider un Molière moribond. D'un point de vue stylistique, cette relation intertextuelle montre que l'esthétique de Wilson et ses mondes surréalistes contiennent des associations culturelles et intellectuelles, qui permettent de procéder à des interprétations. 


\section{«TOUT LEUR ART EST PURE GRIMACE » 1 LE PERSONNAGE DU MÉDECIN DANS L'GEUVRE DE WILSON}

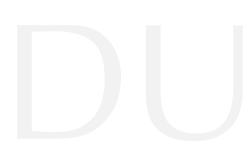

Traduit de I'anglais par Jean-Pierre Vidal

SHARON ARONSON-LEHAVI

Commençons par la fin. À la toute fin de la vidéo La Mort de Molière, après le générique, après la mort de Molière et son enfouissement dans une tombe envahie par les flammes de l'enfer, il reste encore une dernière séquence. Le médecin en costume moderne, qui est déjà apparu trois fois comme personnage marqué par l'aliénation, apparaît une fois de plus, en train de fumer une cigarette avec manifestement un grand plaisir. D'abord il regarde une religieuse assise près de la fenêtre; elle détourne son visage de lui avec une certaine brusquerie; alors, vu de son profil gauche, il rejette la fumée de sa bouche et regarde la caméra avec une expression cynique. Cette conclusion rappelle en fait le début du Dom Juan de Molière où l'on voit Sganarelle, «tenant une tabatière», dire: "Quoi que puisse dire Aristote et toute la Philosophie, il n'est rien d'égal au tabac» (I, 1). L'attitude humoristique de Sganarelle va avec l'expression du visage du docteur et ajoute une perspective à la fois ironique et plus légère à l'atmosphère sérieuse de la vidéo. Sganarelle prisait, mais dans la vidéo c'est une moderne cigarette qui vient symboliser de façon ambiguë le plaisir défendu, tout comme le costume du docteur est moderne: blouse blanche, chaussures blanches, cravate noire, lunettes et cheveux gris coupés court, ce qui permet de reconnaître facilement une icône sociale appartenant à la réalité $\mathrm{du} \mathrm{XX}^{\mathrm{e}}$ siècle.

Montrer un docteur moderne instaure inévitablement une analogie entre la mort de Molière et les attitudes contemporaines à l'égard de la maladie, de la mort et de la médecine. La confiance rationaliste moderne en la science et en la médecine, qui attribue aux médecins le pouvoir de sauver les malades et les mourants, devient ridicule quand le sérieux du docteur devient en fait l'écho lointain du sot de Molière: Sganarelle. En analysant l'image du docteur dans la vidéo, j'entends montrer qu'on peut assurément y voir un lien avec Sganarelle et que sa présence convoque ainsi d'importantes significations thématiques rattachées à la médecine, à l'art et à la mort, qui viennent démentir l'impression d'opacité que donne l'œuvre dans son ensemble. Je soutiendrai aussi que l'image du médecin représente un exemple privilégié de la façon dont les images de Wilson prennent toute leur charge intellectuelle au point d'admettre plusieurs interprétations possibles, toutes également vraisemblables. En d'autres mots, 
parallèlement aux images intuitives et au style esthétique mais aussi onirique qui caractérisent la vidéo, on peut effectivement y trouver des idées et des significations textuelles et intertextuelles claires ${ }^{2}$. Ces idées se rattachent à toutes les autres composantes non thématiques de la pièce qui semblent créées (et aussi absorbées) intuitivement et inconsciemment, et elles leur ajoutent une dimension ironique. L'idée fort répandue selon laquelle l'art de Wilson est un collage d'images $^{3}$ s'applique aussi fort bien à cette œuvre. Les contrastes internes qui lient les diverses images agissent comme révélateurs réciproques de façon inattendue; de plus, cette technique permet à Wilson de fusionner dans chaque image prise isolément des éléments conscients/thématiques et inconscients/ intuitifs, exactement comme les images apparaissent dans un rêve ou dans un flux de conscience. En conséquence, certaines des images, comme celle du médecin, suscitent des interprétations logiques et ironiques tout en affectant le spectateur de façon inconsciente.

\section{SGANARELLE, Molière ET WiLSON}

De façon assez surprenante, la cigarette n'est pas la seule allusion à Sganarelle faite par le médecin au cours de la vidéo. Au contraire, presque toutes les apparitions du médecin tout au long de l'œuvre évoquent tel ou tel comportement de Sganarelle dans une pièce de Molière. Le costume moderne du médecin représente sans contredit une des indications principales de cette similarité. Dans sa critique généralisée et très appuyée de la médecine et des médecins, Molière déguise souvent Sganarelle en médecin et il s'agit toujours d'une caricature ridicule ${ }^{4}$. L'habitude qu'a Sganarelle de revêtir un habit de médecin, soulignant l'insignifiance de la profession, peut donc expliquer pourquoi le médecin de la vidéo est le seul à porter des vêtements modernes, imitant ainsi Sganarelle lorsqu'il se déguise.

Mais ce n'est pas seulement l'accoutrement du médecin qui crée la similarité. Le fait qu'il fume à la fin de la vidéo, après le déroulement du générique final, quand tout est fini, exactement comme dans le cliché "fumer après l'amour», symbolise deux fins: celle de l'œuvre d'art et celle de la vie de Molière. Le fait que, de tous les personnages que l'on voit dans l'œuvre, ce soit le médecin qui fume à la fin doit bien signifier quelque chose quant au rôle qu'il a joué dans la mort de Molière. Ironiquement, comme Sganarelle déguisé en médecin dans le troisième acte de Dom Juan parle à son hérétique de maître d'un patient que les médecins ont sauvé en le tuant, il semble que le docteur de la vidéo ait "réussi" de la même façon:

Sganarelle: Il y avait un homme qui, depuis six jours, était à

l'agonie; on ne savait plus que lui ordonner, et tous les remèdes ne faisaient rien; on s'avisa à la fin de lui donner de l'émétique. Dom Juan: Il réchappa, n'est-ce pas?

Sganarelle: Non, il mourut.

Dom Juan: L'effet est admirable.

Sganarelle: Comment? Il y avait six jours entiers qu'il ne pouvait mourir, et cela le fit mourir tout d'un coup. Voulezvous rien de plus efficace? (III, 1)

S'ajoutant à l'accusation que les médecins en fait tuent leurs patients, les mots de Dom Juan à Sganarelle, quelques répliques plus loin, à l'effet que la médecine «est une des grandes erreurs de l'humanité", cités également par Müller au début de la «scène du docteur» de la vidéo, deviennent ironiquement vrais par cette métonymie. La phrase suivante de Müller, «Molière se meurt», récitée sans cesse tout au long de la vidéo comme une façon de marquer le rythme et d'assurer thématiquement la mort de Molière, prouve la véracité des déclarations de Dom Juan. Non seulement le médecin s'avère incapable de guérir Molière, mais il ne peut en fait que l'«aider» à mourir. L'idée d'accuser le médecin de la mort de son patient se trouve soulignée dans la vidéo par l'adjonction à la scène du médecin d'un extrait de la nouvelle de Kafka, Un Médecin de campagne. Dans cette histoire, racontée du point de vue du médecin lui-même, celui-ci ne parvient pas à sauver le garçon de la mort et se montre frustré de l'attitude des gens du pays qui «exigent toujours l'impossible du médecin». Cette conjonction du médecin de Kafka, de Sganarelle l'imposteur et du 
médecin de la vidéo nous montre trois médecins qui se trouvent liés à la mort d'un patient plutôt qu'à sa guérison. Ce genre de caractérisation contraste fortement avec le statut du docteur dans la culture et l'art populaires, où les médecins sont généralement dépeints comme efficaces, bienveillants, savants et pleins d'autorité, doués du pouvoir presque magique de dominer et de contrôler la vie des autres. On les y représente comme des figures omnipotentes qui ont le pouvoir de sauver des vies contre toute attente ${ }^{5}$. L'attitude opposée de Wilson à l'endroit du médecin, qui consiste au contraire à révéler son impuissance, attitude probablement influencée par celle de Molière, atteint son point culminant dans la première "scène du médecin", qui est aussi la plus importante.

Dans cette scène, le médecin est d'abord montré à droite, près du lit de Molière et regardant étrangement le patient. Après quelques plans rapides des religieuses et de Molière portant un mouchoir à sa bouche, le médecin réapparaît, mais cette fois à gauche et marchant lentement dans une direction opposée à celle dans laquelle il marchait auparavant. On peut en déduire que cette fois il va vers le fauteuil vide de Molière puisque les fenêtres sont vues cette fois sur sa droite. S'éloigner ainsi du patient représente ironiquement l'exact opposé de ce que fait le médecin de campagne de Kafka avec son patient dans le texte récité en voix off. Il demande, en effet, de coucher à côté du jeune garçon malade ${ }^{6}$. Mais même ainsi, il ne peut qu'admirer érotiquement la blessure au corps du garçon; il appelle cette blessure «cette fleur dans ton flanc» et laisse le garçon mourir lentement mais sûrement, entouré de sa famille, exactement comme Molière. Quand le médecin de la vidéo atteint le fauteuil, il le frappe violemment, là encore en contraste avec la voix douce lisant la description que fait Kafka de la blessure du garçon et la musique élégiaque de Glass en fond sonore. Le fauteuil que l'on frappe avec fracas apparait pour la première fois à l'image dans cette scène, mais le bruit qu'il fait a déjà été entendu deux fois auparavant, en accompagnement sonore de l'image du fauteuil vide. Le bruit désormais familier rattache ainsi rétroactivement le fauteuil vide qui symbolise l'absence de Molière à la réaction agressive du médecin devant son patient à l'agonie. On remarquera que ce geste du médecin apparaitra une autre fois dans la vidéo comme un flash-back, immédiatement après la courte scène dans laquelle il essaie nerveusement d'ouvrir la cage de verre qui contient le fauteuil vide de Molière. Cette fois, on voit et on entend le fauteuil frappé accompagné d'un autre bruit qu'on a déjà entendu auparavant: celui du verre brisé. Tandis que sur le plan sonore, tout se passe comme si le coup avait brisé la vitre, permettant ainsi au médecin d'entrevoir l'inconnu, visuellement la cage de verre reste intacte. Au contraire, le docteur apparaît encore impuissant et ridicule dans son effort désespéré de pénétrer le vide laissé par Molière après sa mort.

Après la scène montrant le médecin frappant le fauteuil pour la première fois, une des religieuses lui présente le pot d'urine de Molière. Ce geste donne l'autre clé de la relation intertextuelle entre le médecin et Sganarelle et ajoute un autre niveau interprétatif au personnage du médecin. L'action de boire l'urine, comme le fait le médecin, lentement, bruyamment et goulûment, semblerait indéchiffrable et plutôt étrange si on ne la rapprochait pas d'une des premières pièces de Molière mettant en scène un médecin, la farce en un acte intitulée Le Médecin volant (1658). Dans cette pièce, Sganarelle est aussi déguisé en médecin et tente de découvrir ce qui ne va pas chez Lucille, la jeune fille souffrant du mal d'amour. Afin de pouvoir poser un diagnostic, il insiste pour boire son urine; il la boit, en apprécie le goût et en redemande:

Gorgibus: Oui-da; Sabine, vite allez quérir de l'urine de ma

fille. Monsieur le médecin, j'ai grand peur qu'elle ne meure.

Sganarelle: Ah! qu'elle s'en garde bien! il ne faut pas qu'elle

s'amuse à se laisser mourir sans l'ordonnance du médecin. Voilà

de l'urine qui marque grande chaleur, grande inflammation

dans les intestins: elle n'est pas mauvaise pourtant.

Gorgibus: Hé quoi? Monsieur, vous l'avalez?

Sganarelle: Ne vous étonnez pas de cela; les médecins,

d'ordinaire, se contentent de la regarder; mais moi, qui suis un 
médecin hors du commun, je l'avale, parce qu'avec le goût je discerne bien mieux la cause et les suites de la maladie. Mais, à vous dire la vérité, il y en avait trop peu pour asseoir un bon jugement: qu'on la fasse encore pisser.

(Le Médecin volant, IV; c'est moi qui souligne)

Une fois de plus, l'image vidéographique, qui semble déraisonnable et dégoûtante, est en fait une citation intertextuelle recherchée du valet moliéresque tel qu'il apparaît dans cette première farce médicale. Goûter l'urine était une composante bien connue de l'art du diagnostic de tout médecin jusqu'à la fin du XIX' siècle: on s'en servait par exemple pour diagnostiquer le diabète si le goût en était sucré. Mais on ne disposait d'aucun traitement efficace 7 . La façon dont le médecin de la vidéo boit l'urine, dans un complet détachement à l'égard de son patient, comme s'il s'agissait d'un geste purement mécanique, souligne la prétention de la profession médicale. En outre, la scène devient grotesque à cause de la façon dont il l'avale, avec passion, exactement comme le fait Sganarelle, alors que les vrais médecins se contentaient de la goûter du bout de la langue. De toute façon, cette technique ne servait à rien du temps de Molière et donc ici aussi elle ne change pas grand chose, même si pour Sganarelle elle «marche», comme marcherait n'importe quel traitement appliqué à tout hasard.

Le fait qu'il boive l'urine, fume une cigarette et porte un costume moderne nous permet incontestablement d'interpréter le médecin de la vidéo comme une version un peu tordue de Sganarelle déguisé. Par delà la perspective comique amenée par cette métonymie et les interprétations ironiques qu'elle convoque et qui soulignent l'impuissance du médecin et l'absurdité de la foi en la médecine et en la science, on doit considérer un autre facteur. La relation qu'entretenait Molière avec Sganarelle était plus que celle qui lie un dramaturge à son personnage. Sganarelle a pratiquement accompagné Molière tout au long de sa vie: non seulement Molière l'a-t-il inventé, mais il a aussi effectivement tenu le rôle de Sganarelle dans toutes les pièces où celui-ci apparaît, souvent déguisé en «faux-médecin ${ }^{8}$. Ceci veut dire que le médecin de la vidéo peut en fait être vu non seulement comme une métamorphose de Sganarelle, mais aussi comme Molière l'auteur déguisé en son personnage, qui lui-même est déguisé en médecin, un médecin qui finalement boucle la boucle en «soignant» le dramaturge agonisant. Ironiquement, à la fin, Molière doit s'affronter lui-même, il doit affronter sa propre critique de la profession médicale, espérant désespérément et en vain que son iconoclasme s'avère sans fondement. Au lieu de cela, chacune de ses plaisanteries devient non seulement juste mais tourne au tragique.

Cette situation imaginaire dans laquelle la figure du médecin apparaît comme un combiné impossible de personnages fictifs et de personnages historiques est comme la structure floue d'un rêve. En chargeant d'une telle intensité l'image du médecin et en créant en même temps une persona aussi aliénée, Wilson trouve effectivement moyen d'illustrer les pensées et les angoisses qui occupent l'esprit d'un homme en train de mourir. Le cliché bien connu qui veut que «la vie entière d'un mourant se déroule en un éclair devant ses yeux» se trouve matérialisé, dans la vidéo, par la combinaison de diverses situations et de divers personnages qui firent partie de la vie de Molière et qui eurent un impact sur lui, simultanément et à différents niveaux de sa conscience.

Mais ces cercles concentriques se compliquent encore du fait que dans la vidéo le rôle de Molière est tenu par Wilson. Ce genre de parallélisme est particulièrement intéressant: Molière a inventé Sganarelle et lui a littéralement insufflé la vie en jouant son rôle; et Wilson fait la même chose pour le personnage de Molière. Molière devient ainsi un personnage de fiction plutôt qu'un personnage historique. Le Molière du portrait montré au début de la vidéo, ou signifié par son fauteuil vide trônant à la Comédie-Française, devient en lui-même un texte. C'est potentiellement ce qui va également arriver à Wilson après sa mort, si jamais un autre metteur en scène joue son personnage et le transforme ainsi, de personne réelle et historique qu'il a été, en 
personnage fictif mais éternel. La frontière entre les gens réels, les personnages historiques morts et les personnages de fiction - parfaitement floue sur le plan de la logique - devient parfaitement cohérente au point de vue artistique. C'est ainsi du moins qu'on interprète souvent la formule de Derrida selon laquelle «rien n'échappe au texte»?.

En conclusion, le personnage du docteur dans la vidéo forme non seulement une image multiple, mais il pourrait aussi servir de clé pour pénétrer cette œuvre complexe et compliquée. Son apparition à la fin, après la mort de Molière, est, encore une fois, semblable à l'apparition de Sganarelle à la fin de Dom Juan, pleurant sur ses gages restés impayés après la mort de son maitre. Dans la comédie de Molière, Sganarelle, qui s'en tire comme un diable qui sort de sa boîte, célèbre, en apparaissant une dernière fois, le sens de la vie qui anime la société et qui représente le ressort fondamental de la comédie, au sens où Susanne K. Langer définit le rythme comique ${ }^{10}$. Le docteur de la vidéo fait exactement la même chose lors de sa dernière apparition, puisqu'il porte en lui non seulement le personnage éternel de Sganarelle, mais aussi la figure de Molière, et même celle de Wilson.

\section{Notes}

1. Ce sont les paroles de Dom Juan à Sganarelle à propos des médecins, quand celui-ci apparaît en costume de médecin à la scène 1 de l'acte III de Dom Juan.

2. En accord avec la définition que donne Patrice Pavis des œuvres de Wilson comme mise en scène autotextuelle qui «invente un univers scénique cohérent, refermé sur lui-même dans un système esthétique autonome", je pense que les univers autonomes de Wilson contiennent en fait en eux-mêmes des réseaux d'associations de nature culturelle, qui permettent également de rattacher l'œuvre à des perspectives externes. Voir P. Pavis, "Staging the text", Assaph Studies in the Theatre, vol. 13, Tel Aviv, Tel Aviv University, 1977, p. 71.

3. Voir, par exemple, K. Arens, «Robert Wilson: Is Postmodern Performance Possible? „, Theatre Journal, vol. 43, 1991, p. 14-40. 4. Sganarelle s'habille en médecin dans les pièces suivantes: Le Médecin volant, Dom Juan, Le Médecin malgré lui et Monsieur de Pourceaugnac.

5. Voir D. Lupton, «Representations of Medicine, Illness and Disease in Elite and Popular Culture", dans Medicine as Culture, London, Sage Publications, 1994, p. 53

6. Voir F. Kafka, «Un Médecin de campagne», dans Euures complètes, tome II, Paris, Gallimard/NRF, coll. "Bibliothèque de la Pléiade", p. 440-446.

7. Voir, par exemple, A. S. Lyons et R. J. Petrucelli, Medicine: an Illustrated History, New York, Harry N. Abrams Inc., 1978, p. 445.

8. Voir R. W. Herzel, The Original Casting of Moliere's Plays, Ann Arbor, Michigan, University of Michigan Press, 1981.

9. J. Derrida, De la grammatologie, Paris, Éd. de Minuit, 1967, coll. "Critique".

10. S. K. Langer, Feeling and Form, New York, Charles Scribner's Sons, 1953, p. 326-350. 


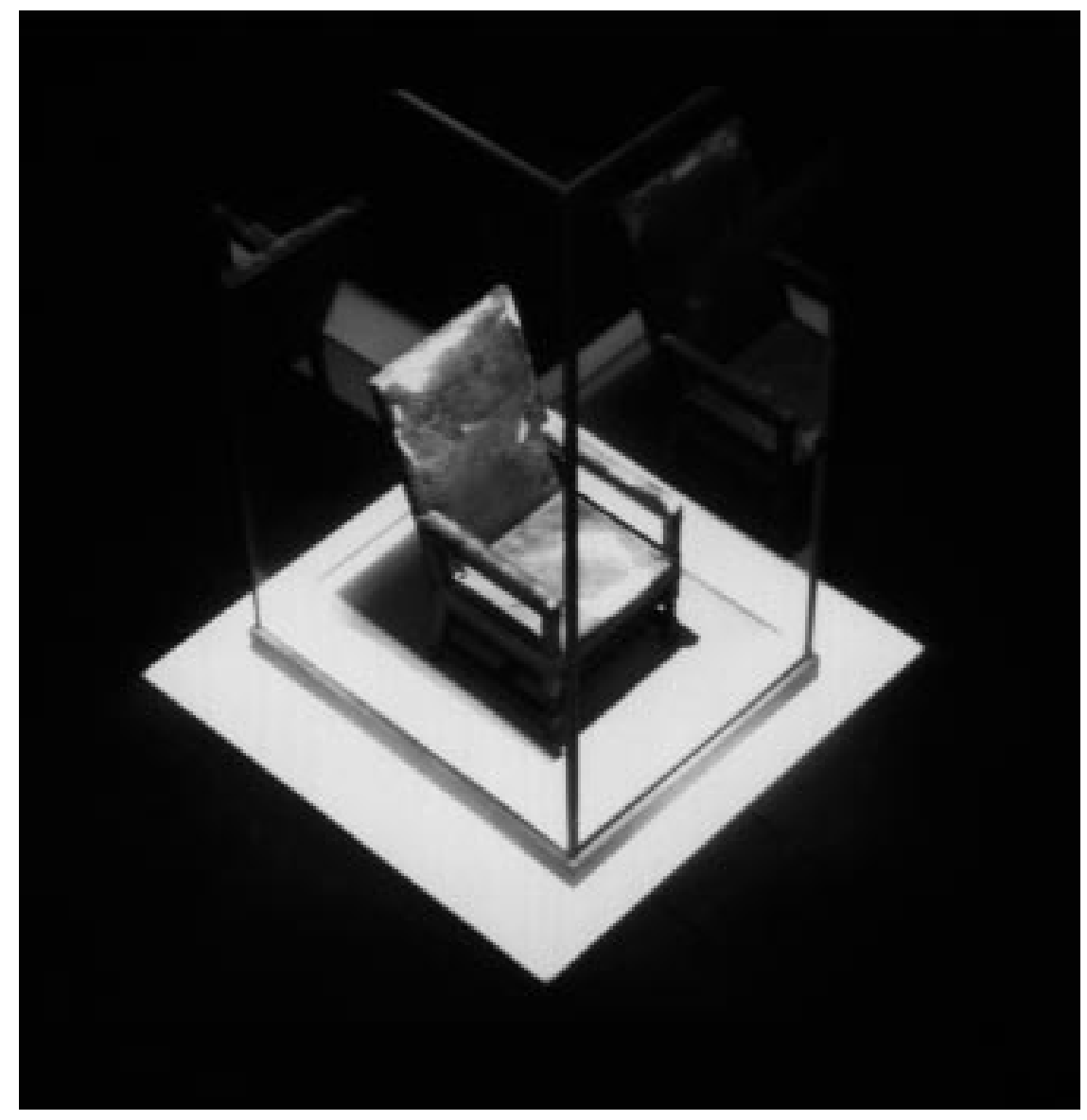

La Mort de Molière de Robert Wilson. Photo INA. 